\title{
El desprendimiento del tiempo sedimentado en el diseño. The detachment of Apuntes para una fenomenología de los objetos ${ }^{1}$ time settling in design. Notes for a phenome- nology of objects
}

Vanegas, J. y Maldonado, L. (2015). El desprendimiento del tiempo sedimentado. Apuntes para un fenomenología de los objetos. Designia, 3 (2), 39-61.

\section{Palabras clave:}

\author{
Prospectiva \\ Key words: \\ Phenomenology, artifact, \\ design, zeitgeist \\ Recibido: 2-Jul- 2015 \\ Aceptado: 15-feb- 2016
}

* PhD en Filosofía. Profesor e investigador titular de la Universidad Autónoma de Manizales. Coordinador del Departamento de Diseño y Arte. Miembro del grupo de investigación Diseño y Complejidad. hovg@autonoma.edu.co

\section{* Magíster en Filosofía. Coordinadora del Programa de Diseño de Modas de la Universidad Autónoma de Manizales. Líder del grupo de investigación Diseño y Complejidad \\ Isuiomar@autonoma.edu.co}

\section{Este artículo hace parte del la} fundamentación del Proyecto Educativo del Programa de Diseño Industrial de la Universidad Autónoma de Manizales.
El futuro me rebota hacia el pasado, éste hacia el presente, de aquí voy otra vez al futuro que me arroja al pasado y éste es otro presente en un eterno girar. José Ortega y Gasset (2006, p. 229)

\section{Resumen:}

Este artículo realiza una reflexión del concepto de objeto u artefacto de diseño desde una perspectiva fenomenológica. Se trata de señalar la constitución temporal de los objetos, presentando su aspecto fantasmal como pasado, así como la experiencia de futuro que cabe a través del ejercicio de proyectación del diseño, pues la constitución del objeto implica la presencia de lo pasado para su nuevo desarrollo y su proyección en el futuro. Se revisa también la transformación hacia su minimalización mediante la abstracción de elementos, la permanencia en el tiempo y su consecuente olvido, perspectiva desde la cual se señala su desvanecimiento en el mundo objetual. Por otra parte, se muestra su surgimiento a partir de las necesidades humanas, sentido que lleva a la intencionalidad de su generación en el mundo desde un sujeto.

\section{Abstract}

This article is a reflection about concept of design object or design artifact from a phenomenological perspective. It tries to note the temporary constitution of objects, presenting his shostly appearance as past, and the experience of the future that is possible through the exercise of projecting of design, since the creation) of the object, implies the presence of the past for new development and its projection in the future. also is reviewed the transformation towards their minimization by abstracting OF elements, the permanence in time and their consequent oblivion, perspective from which is marked its fading in the world of objets. Moreover, its emergence is shown from human needs, meaning that leads to the intentionality of his generation in the world from a subject. 


\begin{abstract}
Algunas de las ideas más fuertes relacionadas con la configuración material del mundo son las siguientes: primero, el acontecimiento de la prospectiva transferido a la materialidad o idealidad del diseño en general, entendida la prospectiva como el diagnóstico y la preparación del instante vivido para acomodar el futuro próximo o cercano.
\end{abstract}

Segundo, la interpretación de los acontecimientos del pasado, inmediato o mediato, plasmados o expresados en la materialidad del mundo y advenidos al presente con nuevas configuraciones, pero con las mismas ideas. En este caso, se traen al ahora los significados más predominantes de lo pretérito, para acomodarlos a las circunstancias del presente. Tercero, lo inmediato que siempre tiene valor en cada época, cuando no en el fluir mismo de la historia. El activismo en la configuración material del mundo es muy frecuente, pues se considera menos difícil, por su exigencia pragmática y su ausencia de fundamentos teóricos.

El diseño navega en el fluir de la temporalidad, pero su trabajo cobra legitimidad en el instante actual, en tanto el advenimiento del pasado y la espera del futuro dan sentido a los productos del presente. En cada configuración material, cuando no imaginaria o ideal, en cada producto del diseño brota una cantidad infinita de ideas. En los objetos resplandece un horizonte de posibles lecturas, por consiguiente, cuando se les actualiza no solo se transforma su estructura material, sino también que su conjunto de ideas cambia cuando se les trae al presente. Algunas de estas ideas 
son características del pasado y otras son sedimentaciones del futuro. Sin embargo, esto es una fantasía de la palabra, pues los objetos no tienen historia, ni futuro; tales declinaciones temporales se dan únicamente en su relación con los sujetos. El fluir temporal de los objetos no se encuentra en ellos sino en la intencionalidad humana, por tal razón, la característica fundamental de los productos del diseño no está dada en ellos sino en los significados que los seres humanos (los diseñadores) les imprimen.

Bajo estos presupuestos, en el instante presente del diseño se fusionan el ahora del pasado y el ahora del futuro, pues lo único que tiene existencia material en el tiempo es el presente, ya inapresable y vaporoso. De esta manera, se puede plantear la siguiente hipótesis: si en los productos del diseño se encuentran encapsulados el tiempo ido y el no sido, entonces en la interpretación de los objetos se encuentra la sedimentación del futuro. El objetivo de esta reflexión es comprender el sentido del futuro en el ejercicio del diseño. Para mostrar tal hipótesis es preciso desarrollar tres puntos: primero, la experiencia del futuro a través del diseño; segundo, la constitución temporal de los objetos de diseño; tercero, el nexo entre los fantasmas de los objetos y los objetos fantasmales.

\section{LA EXPERIENCIA DEL FUTURO A TRAVÉS DEL DISEÑO}

La pregunta inicial en esta reflexión: Les posible tener experiencia de futuro? Más precisamente: ¿puede el diseño generar una experiencia de lo que no ha acontecido en el tiempo? Para responder estos interrogantes es necesario exponer algunos apuntes sobre la experiencia del tiempo en general, en aras de determinar la posibilidad de vivenciar lo no sido del diseño. La evidencia en el fluir de la existencia de la humanidad genera la idea de que lo único existente es el presente, ese instante que nos acompaña en todo momento. Los humanos estamos condenados a vivir en un constante presente, el pasado y el futuro son ideas sin existencia tangible, no existen de hecho y solo tienen valor como nociones encapsuladas en la nada. Al respecto afirma Bachelard (1999): "El tiempo solo tiene una realidad, la del instante. En otras palabras, el tiempo es una realidad afianzada en el instante y suspendida entre dos nadas. No hay duda de que el tiempo podrá renacer, pero antes tendrá que morir" (p. 11). Frente a esta idea de la temporalidad del ya, también se refiere Ortega y Gasset (2006): "El tiempo cósmico solamente es el presente porque el futuro todavía no es y el pasado ya no es. ¿Cómo entonces pasado y futuro siguen siendo parte del tiempo?" (p. 229). No obstante, a pesar 


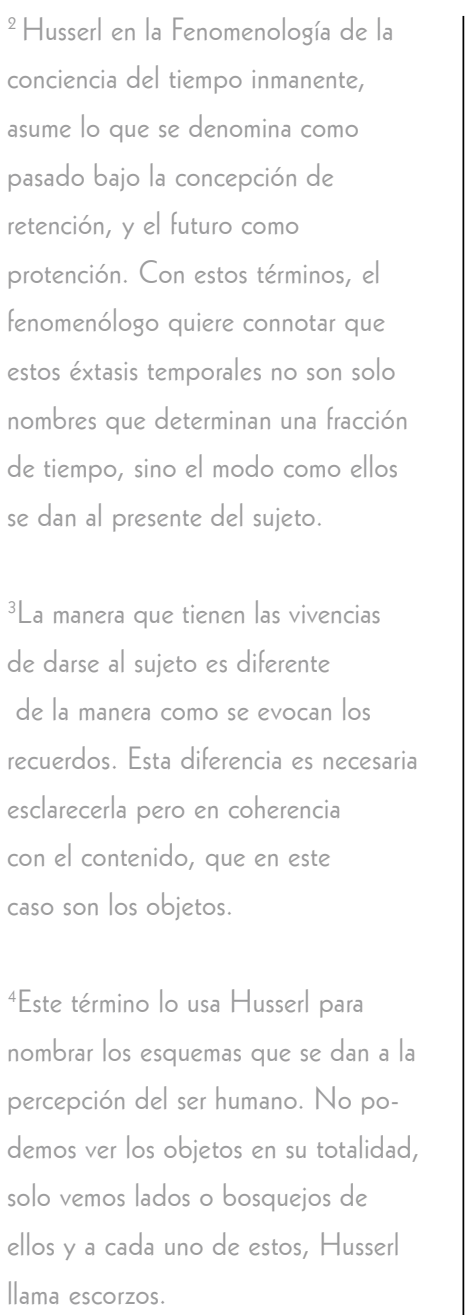

de la desmaterialización del pasado y del futuro en el instante vivido, el ahora conserva elementos de la nada que rodea la vivencia del presente, los cuales pertenecen a los recuerdos, en el caso del pasado, y pueden ser denominados como retención. Asimismo, posee elementos del futuro revelados en el presente: son los contenidos de la espera o la protención, según le llama Husser ${ }^{2}$. Se trata de sedimentaciones aún vivas de los éxtasis del tiempo en el instante vivido.

En coherencia con lo anterior, el tiempo se revela a la experiencia del sujeto en cada uno de los instantes del presente, y estos contienen un momento de rememoración y otro de espera. Esto es, el instante posee de una manera consciente, aunque no siempre intencional, vivencias en forma de recuerdos ${ }^{3}$. El futuro también se hace visible para el pensamiento en el ahora vivido. Esta presencia en lo presente existe como horizonte, como apertura y espera, al ser un elemento profético que aparece en el instante vivido y le imprime un contenido futurista. De esta forma se funden en el instante presente lo sido y lo que puede llegar a ser. En la experiencia del mundo habitamos en dos órdenes temporales, "Es preciso estar en dos órdenes: frente a lo que se ha perdido y en la anticipación de lo que nos espera (Baudrillard \& Nouvel, 2002, p. 27).

El presente es un límite pero a la vez un horizonte, o mejor, es el lugar de donde brotan todos los horizontes posibles. El instante vivido está suspendido en la inmaterialización del pasado y en los fantasmas del futuro. Ahora bien, la experiencia del tiempo como se ha tematizado hasta aquí es eminentemente formal, pues le hace falta el contenido. El tiempo, como el espacio, constituyen la forma y esta reclama un contenido para completar la experiencia. A estos contenidos podemos llamarlos "objetos de la vivencia". En ese instante-límite se da la experiencia de los objetos que aparecen ante el sujeto por medio de los sentidos, incluyen características de los mismos y excluyen aquellos elementos imperceptibles. Los límites de la sintáctica de los objetos no solo están en ellos sino en la incapacidad del ser humano de percibirlos en su totalidad. La percepción humana se brida a los sentidos en forma de escorzos ${ }^{4}$. Pero al ser los objetos bidimensionales o tridimensionales, sus 
dimensiones no son dadas al sujeto por medio de los sentidos sino como síntesis de la conciencia y la razón, es decir, no hacen parte de la experiencia objetual de los seres humanos sino de las inferencias que pueden hacerse en coherencia con los residuos del pasado que quedan en el ahora de la vivencia objetual frente al mundo material.

\section{El mundo físico se presenta en forma} de bocetos y la experiencia acumulada tanto de los recuerdos como de la perspectiva de futuro, adiciona a estos bocetos los contenidos necesarios para hacerlos objetos de las vivencias humanas. Esta idea se revela históricamente cuando se contempla el arte primitivo expresado en la pintura rupestre, datada en aproximadamente 40.000 años A. C. Aquí se revelan los esbozos que los hombres primitivos tenían en el pensamiento, si bien no es posible encontrar propiamente la tridimensionalidad, a diferencia de las pinturas actuales que plasman $\circ$ pretenden dar la idea de la totalidad de los lados, la profundidad de los objetos del mundo (quizá uno de los ejemplos más representativos fue Picasso). Este mismo ejemplo lo podemos reducir en el tiempo cuando se piensa en la génesis de la percepción del ser humano y su duración en el mundo de la vida: un neonato no ve, en sentido estricto, aunque tiene órganos visuales. Su perspectiva del mundo externo es mínima, se va desarrollando y alimentando a medida que tiene más nociones, conceptos y categorías preelaboradas del mundo, las cuales son transferidas a la mente en el transcurso del tiempo. Bajo esta perspectiva, la experiencia de los objetos vivenciales al desnudo, lo que se brinda a los sentidos respecto al diseño de la materia, es solo un fragmento de la misma. La mayoría de las vivencias objetuales están constituidas por los inquilinos del recuerdo y las perspectivas del futuro ancladas al presente de la vivencia del objeto.

Hasta aquí se tienen dos elementos que limitan la existencia de los objetos o artefactos de las vivencias. Primero, solo se dan al sujeto en el instante vivido, quizá en muchos instantes, pero son intermitentes para la percepción y no tienen forma de salir de las fronteras de los presentes vividos. Segundo, no se brindan a los sentidos tal cual son en el mundo externo, sino con los límites que constituyen los sentidos para la percepción del mundo. De esta manera, la constitución de los objetos está 
enmarcada en la función de los sentidos: la imagen, el sonido, el sabor, el olor y la textura conforman la percepción que se hace de ellos. Para los sentidos, no son más que esto. Cómo explotar estas vivencias es una de las tareas del diseño en una actualidad que ha resaltado la imagen y ha reducido, tanto material como virtualmente, el sonido, el rastro, la huella y la piel de los objetos. Vivimos en una cultura de la imagen visual, sin desconocer la importancia de esta para la humanidad. Se han minimizado las otras donaciones que el objeto brinda a los diseñadores. He aquí uno de los retos futuristas del diseño: generar diversas vivencias objetuales para llenar el vacío de los sentidos que ha marcado la modernidad.
Cuando hablamos de objetos de las vivencias, con las características que venimos desarrollando, nos referimos a la individualización de la "realidad" por medio de la individualización de la sensación. De esta manera, la determinación de una fracción de la realidad solo es posible a partir de la determinación de una fracción de la sensación. Los límites temporales y espaciales de esta última son los límites de la realidad y al continente que se delimita con ella se le denomina objeto. Ahora bien, como puede inferirse, los límites que determinan la existencia del objeto no están en la realidad fáctica sino en la mente humana. Por lo tanto, la experiencia de los objetos se da mediante el advenimiento de los límites que los seres humanos han generado para darles vida. De esta reflexión surge la pregunta: ¿cuál es el origen de los límites que determinan los objetos de las vivencias? Por una parte podemos responder, aunque esto es hipotético, que dichos límites parten de las necesidades básicas de los sujetos en el mundo práctico: cuando alguien necesita transportar líquido para suplir su necesidad de sed, limita una fracción de la naturaleza física en forma de contenedor. Este "uso" genera en la sensación unos límites que dan forma a objetos como ánforas, vasos, frascos o cualquier contenedor. Por otra parte, podemos pensar en objetos estéticos (pinturas, esculturas) en los cuales el origen del límite no surge de una necesidad pragmática sino anímica, emocional.

De esta manera, podemos pensar en experiencias funcionales y experiencias estéticas de los objetos. En las primeras, los seres humanos se prolongan en la naturaleza física, se dan corporalmente para sobrevivir, deben adaptar el mundo a sus circunstancias y hacerlo un hábitat que les permita suplir sus necesidades. Los objetos manipulables, como contenedores, lascas o cuchillos rudimentarios, trajes incipientes para protección del frío, sillas, camas, baños, facilitan la sobrevivencia del sujeto corporal, el cual se extiende a las cavernas, al nicho o al nido, pues requiere un sitio de protección. "La casa no solo dispensa en el interior calor y comodidad, sino que con respecto al interior le da al hombre firmeza y fuerza para prevalecer contra el mundo" (Bollnow, 1969, p. 126). La casa es el mundo en miniatura generado por el ser humano como producto de su impotencia frente a la magnitud del mundo. Con esta protección asegurada se extiende al pueblo o la ciudad, con sus 
edificaciones imponentes, lo mismo que a las carreteras y de aquí al país y más allá de sus fronteras. Los objetos surgen de la experiencia corporal humana de acuerdo con sus necesidades.

Sin embargo, y esta es la segunda experiencia, los seres humanos no quedan satisfechos con suplir sus necesidades de supervivencia. No les es suficiente con comer, también condimentan los alimentos pues más que suplir una necesidad, buscan satisfacer el gusto. Por ello, crean un mundo de significados estéticos que no solo les permite sobrevivir, sino también hacerlo de la mejor manera. Hombres y mujeres idean objetos que llenan los sentimientos de gozo. Así, el mundo está lleno de maravillas generadoras de satisfacción para el espíritu, como también de artefactos resultantes del diseño. Aquí aparece la gran paradoja entre la estética y la función en el diseño: entre más estética tiene un producto menos función posee y por el contario, entre más función menos estética. El punto preciso es el medio perfecto entre utilidad y belleza.

Tenemos entonces que la experiencia de los objetos vivenciales en el instante presente se da mediante la limitación de los sentidos en coherencia con la realidad, lo cual depende de la función o la satisfacción. Los objetos son fracciones de la realidad, sin embargo, la reducción sensorial no termina aquí. La misma capacidad sensual que aplicamos a la realidad para particularizar los objetos la ponemos, y de hecho la empleamos, en la experiencia de estos. Al tomarlos como una totalidad real, física o abstracta, los descomponemos con los sentidos en cada uno de los elementos que conforman su "sintaxis": color, forma, extensión, entre otros. Así como una casa - un objeto a escala mayor - tiene puertas, paredes, ventanas, cocina, habitaciones y baños, los objetos en general poseen partes que el sujeto, en un acto de conciencia, relaciona con el todo y este a su vez con las partes. La vivencia del objeto posibilita incorporar en la conciencia cada uno de estos componentes. Es más, la objetividad misma se crea como unidad de conciencia de cada una de las partes respecto a la identidad del todo, como expresa Husserl (1959, p. 116): "La objetividad presupone una conciencia de unidad, una conciencia de identidad. Aquí consideramos el contenido de cualquier protoimpresión como un algo-mismo".

De aquí surgen las preguntas obligadas para el diseñador: ¿cómo se relaciona la parte con el todo y el todo con las partes? ¿Cómo generar apariencias armoniosas, trágicas, cómicas, caóticas, pírricas, en la totalidad del objeto? Es decir, ¿cómo producir mensajes intencionales, tales como confianza y poder, en los artefactos? ¿Cuáles son los puntos nodales, físicos o abstractos, que unen cada una de las partes y a estas con el todo? ¿Qué magia acontece en la unión entre ellas para expresar un concepto del objeto? ¿Cómo participan las partes del pasado con aquellas que evocan el futuro? Frente a estos eventos, de acuerdo con la teoría fenomenológica, pueden 
existir objetos dependientes de la experiencia entre las partes, en tanto otros son independientes. Husserl (1995) en las Investigaciones Lógicas II, los denomina simples y complejos: "no necesita acaso todo objeto tener partes; de donde resulta la división ideal de los objetos en simples y compuestos" (p. 337).

Es propio, entonces, considerar que los objetos en "sí mismos" tienen una sintaxis y como tal poseen partes que, a su vez, están unidas a su totalidad. En este sentido, hay cualidades o partes indispensables, pertenecientes a la identidad misma del objeto, como por ejemplo la extensión de una mesa, sin la cual esta deja de ser tal; la extensión es dependiente de la mesa y constituye la esencia de la misma. En un intento de generalización es preciso advertir que los objetos poseen componentes dependientes $e$ independientes, pues también existen elementos sin los que un objeto no pierde identidad, tales como el color y en algunos casos la forma. En estas partes efímeras el diseñador puede intervenir al poner una intencionalidad mediante un nuevo significado. Una característica de los diseñadores es la habilidad para depositar intencionalidades en los objetos y específicamente, para variar sus elementos independientes.

Ahora bien, esas intencionalidades transferidas a elementos materiales pueden tener significados rememorados, es decir, del pasado, o también del futuro. El primero no es difícil, sin decir con ello que sea un trabajo sencillo. No obstante, traer de la inexistencia en el tiempo elementos significativos para incorporarlos en los objetos es más complicado, al ser el futuro un detonante que estalla en la conciencia del diseñador, quien recoge las esquirlas lanzadas y las actualiza, las deposita en el presente. Otro interrogante se refiere a las características o identidades que poseen dichas esquirlas para guiar al diseñador en esa búsqueda futurista. En general, todos los objetos están diseñados para el presente y si este es un instante con un ahora retensivo y un ahora protensivo, según se definió previamente, entonces todos ellos, para vivir en el instante, deben contener partes proféticas que inciten a la espera de lo no sido en el tiempo y el espacio. Esto es, todos poseen señales utópicas que conducen a sus usuarios a proyectarse en el futuro. Basta decir, por ahora, que en los objetos resplandece un conjunto infinito de ideas, y estas, en últimas, les confieren su identidad. Así lo vislumbraron Baudrillard y Nouvel (2002, p. 10) en referencia a las torres gemelas: "Esas dos torres parecen dos bandas perforadas, hoy en día diríamos que se clonan una de la otra, que están ya en la clonación". ¿Eran una anticipación de nuestro tiempo? Entonces, Lel arquitecto no está en la realidad sino en la ficción de una sociedad, en la ilusión anticipadora?.

En cuanto a los objetos simples o no compuestos, esta idea resulta un poco oscura. ¿Cómo pensar un objeto sin partes? La unicidad de las características, la simplicidad, no es fácil de concebir en el pensamiento. Sin embargo, partiendo de un acto de fe, podemos considerar que el sujeto siempre tiende a la particularidad, a despresar, de 
entre más simples más limpios y entre más limpios menos partes. Vivimos en una época en que se les desmaterializa. En un conversatorio entre Baudrillard y Nouvel (2002), el primero manifiesta:

tal manera que la idea de color, por ejemplo, puede existir independiente del objeto que la porta. No obstante, sin llegar a extremos, es oportuno acudir a ejemplos más terrenales como los objetos geométricos (un círculo, un cuadrado, un triángulo). En cuanto a objetos materiales, es posible pensar en un balín, que sin entrar en una reflexión profunda, transmite una existencia simple, sus partes son muy pocas o incluso ninguna, de hecho, nos llega la percepción de una sola parte. La experiencia de este tipo de objetos aparentemente es más sencilla, pero si pensamos en la actualidad, la disminución de las partes es una moda que se impone. Más aun, esa limpieza hace referencia al despojo de todas o algunas de las partes: los objetos,
Lo mejor, acercarse más al corazón analítico de las cosas, es decir, querer develar el interior de la propia materia, hasta las partículas que, en un cierto momento, son completamente invisibles, nos conduce efectivamente a la inmaterialidad, o en todo caso, a algo que no es más representable: partícula, molécula. (p. 53).

Bajo este panorama, la vivencia de los objetos conduce a la experiencia del futuro, ya que en su momento presente se da la particularización de los sentidos y esto se logra a través de la conciencia del instante vivido, el cual contiene un ahora prospectivo y revela la existencia de lo no sido. En la individualización de la realidad y de las partes el futuro se revela como un fantasma al objeto. En el contexto donde habitan los productos del diseño, en la realidad física o abstracta, lo mismo que en las conexiones de las partes del interior en relación con el todo, la identidad se enuncia en la duración en el tiempo. Los objetos no tienen tiempo, ni historia, son cerrados al tiempo, como afirma el creador de la fenomenología: "las cosas materiales están exclusivamente condicionadas desde fuera y no están condicionadas por su propio pasado; son realidades sin historia" (Husserl, 1995, p. 176). Sin embargo, los objetos son temporales: "Bajo el concepto 'objetos temporales en sentido específico' comprendemos los objetos que no son solo unidades en el tiempo, sino que contienen la extensión temporal también en sí mismos" (Husserl. 1959, p. 69). De esta manera, la identidad no está directamente en el objeto sino en su duración y esta habita en la percepción humana. Esa duración garantiza una identidad que, como también ocurre con la durabilidad y la idealidad, solo tienen legitimidad en la conciencia humana. Por ello, a continuación veremos cómo se constituyen los objetos en la reflexión. 


\section{LA CONSTITUCIÓN}

\section{TEMPORAL DE LOS}

\section{OBJETOS DE DISEÑO}

\section{Cuando pensamos en el papel} desempeñado por un diseñador al enfrentar la generación de un producto, se dice que debe haber una idea o unas ideas. El proceso, en últimas, consiste en darles materia, materializarlas, encarnarlas, hacer con las manos lo concebido con el pensamiento. En el lenguaje de los diseñadores se afirma que si lo hecho con las manos sorprende, es decir, mejora lo ideado - si la materialidad supera la imaginación - entonces se ha logrado un muy buen producto. De tal modo, los productos están recubiertos por las ideas que se les incorporan, y en sí mismos, cargan con ideas para ser transmitidas. En todo objeto coexiste la subjetividad humana pues son "los elementos en el mundo de la vida que tienen un significado, producto de la intencionalidad de los sujetos, son producto del insenio humano, por ello en todo objeto encontramos elementos que expresan la subjetividad" (Vanegas. 2008, p. 53). En ellos se deposita una intencionalidad que se expresa en cada uno de sus significados. Incluso, la significación misma, la "semántica objetual" no está directamente en el objeto sino en la conciencia humana, en las palabras. Hace parte de las características de las ideas y no de los objetos materiales.

Existe, entonces, una relación bidireccional entre la idea del objeto y este en sentido fáctico, la cual se fusiona para generar los productos de diseño. El objeto material cobra existencia en la medida que resplandezca un conjunto de ideas y estas se reflejan en su misma existencia. Sin embargo, no se encuentran específicamente en esa materialidad, sino en la conexión con los sujetos y en las ideas que predominan en estos. Piénsese si trasladamos del tiempo presente al pasado, por ejemplo al medioevo, un celular de última tecnología. ¿Qué dirían los habitantes de esa época al enfrentarse a tal artefacto? El objeto no habla solo, es necesaria su relación con la percepción y por supuesto, el contexto. Su significado está en las ideas y no solo en su carne. El mundo, en general, se constituye en la correlación, siempre nos dirigimos a objetos ideales y aun aquellos materiales evocan la idealidad. Al respecto Levinas (2004) reflexiona:

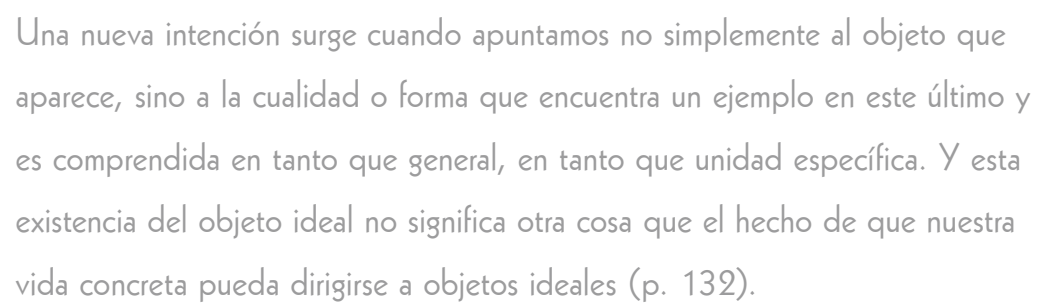


El objeto material es influenciado por las ideas. Estas, en tanto significados, dan identidad a las cosas como objetos y poseen elementos abstraídos de la materialidad. Sin embargo, no son solo acontecimientos abstractos del pensamiento. Al estilo Platón, constituyen la morfología misma de las cosas. Así lo dice Zubiri (1944): "Idea no significa primariamente, como hoy, un acto mental, ni el contenido de un acto mental, sino el conjunto de estos rasgos fisonómicos o característicos de lo que una cosa es. Algo que está en la cosa, sus propios rasgos" (p. 46). En coherencia con esta perspectiva, las ideas no son como las personas del mundo cotidiano las conciben, o no son solo esto, pues son más que el aparecer y el desaparecer de los bosquejos de las cosas en el pensamiento. Son la determinación de la forma de los objetos. Hacen que una silla no sea una cama, o en algunos casos, son las determinaciones formales que llevan a un sofá-cama a ser tal. Son homologables a las formas, lo cual es totalmente válido en cuanto no veamos en los objetos solo su aspecto material sino las ideas que los diseñadores o los seres humanos en general impregnan en ellos. Las ideas caminan en el fluir del tiempo, se han desplegado en el mundo desde que los primitivos crearon el primer artefacto, ya fuera una lasca, una punta de lanza, un contenedor o una pintura. En ese momento empezaron a brotar de la mente y se materializaron en el mundo físico.

Ahora bien, las ideas están encapsuladas en teorías (teo-teros, teorêin) que migran de generación en generación, si bien no de forma estática (cada generación imprime a ellas, es decir a las teorías, ciertas cualidades de acuerdo con las necesidades funcionales y estéticas). Las ideas se van actualizando materialmente en cada advenimiento de lo sido en el ahora vivido, en otras palabras, se van haciendo objetos (no todas, muchas se quedan en el pensamiento y nunca salen de la piel de la subjetividad). Pero las que salen y brotan se hacen visibles a los ojos de la humanidad. En este sentido, el papel del diseño es proporcionar una epifanía al dar materialidad a la forma emanada del pensamiento y legitimada en el mundo de la percepción. Así, la constitución del objeto recoge los acontecimientos objetuales del pasado y actualiza las ideas en el mundo de la vida material: "Manifiestamente en todas estas formas de constitución de objetos nos remontamos a objetos que ya no remiten a objetos predados del tipo de los que primigeniamente se originan mediante cualesquiera espontaneidades, teóricas, valorativas, prácticas" (Husserl. 1984, p. 46).

El conjunto de ideas -o teorías- que determina el concepto de un objeto, puede ser de varias clases en coherencia con la época, el sitio, la región, las necesidades exigidas por las circunstancias. Los objetos tienen plasmadas las improntas de las ideas que los originaron. En ellos pueden leerse conceptos científicos, como resultado de las teorías de la física, la química, la ergonomía, la antropología, la sociología y aun de la psicología. Estas teorías con fundamentos científicos migran del pensamiento del diseñador a la materialidad. Ejemplos de este tipo los tenemos en los productos tecnológicos y las herramientas de trabajo. Aun así, no siempre las ideas que resplandecen en los objetos indican este tipo de teorías. Existen otros que reflejan ideas pre-científicas, fundamentadas en creencias, hábitos, costumbres. Así sucede con algunos productos artesanales, surgidos de nociones sin bases contrastables 
mediante métodos científicos. Esto no significa que carezcan de importancia, solo poseen otro tipo de lenguaje. Aquí queda abierta la pregunta por la formación del diseñador, pues cuando indagamos por las intencionalidades reflejadas en los productos podemos determinar la escuela detrás de ellos, lo mismo que la rigurosidad científica y la recurrencia a factores pre-científicos.

En cuanto al tiempo, hay objetos que se sacan de la historia y se reivindican en la actualidad, caminan en el pasado y se actualizan en los presentes. Así la mayoría de ideas que brotan de ellos evoca un momento pretérito, la actualidad siempre obliga a generar ideas que les dan el sello del presente. Aunque sean de otro tiempo en su actualización, cobran valor del ahora vivido. El contexto excluye los objetos del tiempo sido y los obliga a quedarse en su época. La distancia de la existencia de los objetos y sus circunstancias, hace que aquellos del pasado se constituyan con más o menos fuerza en el presente. Así como algunos se olvidan en el fondo del pasado, otros siguen vivos sin importar lo distante de su surgimiento. En lo estético y en su carácter museológico, empiezan a cobrar valores diferentes a los que inicialmente tenían. La historia les imprime un valor distinto al original. En realidad, todo objeto dado en el presente a los sentidos tiene predecesores, como protoobjetos. "Aquí en el puro dato de sensación tropezamos con una predación que se encuentra como objeto aun antes de la constitución del objeto" (Husserl, 1984, p. 52).

En cuanto a los objetos del futuro, según se dijo, su única existencia se da en el presente como espera, proyección o prospectiva. Lo no sido aparece en el ahora como una utopía pero con grandes probabilidades de acontecer en la medida que se trata de una propedéutica del futuro. Es decir, en el presente coexiste el futuro como propensión y esto se deja ver en los objetos. Lo venidero está dado en el presente, en donde se fabrica el por venir. Las películas futuristas tienen la propiedad de elaborar objetos que dan la apariencia de lo no sido. La sensación que estos deben generar en los usuarios es la de habitar un mundo que no ha tenido realidad pero muy probablemente la tenga. En los objetos de la ciencia-ficción resplandecen ideas que se desprenden del futuro. Bajo este panorama, todo instante vivido contiene ideas del futuro, lejano o próximo. Si las vivencias tienen algo vivenciado, es decir, un objeto, un producto de diseño, entonces los objetos poseen en sí mismos ideas de futuro y una duración en coherencia con la duración de la percepción: "En efecto es evidente que la percepción de la duración, en cuanto tal, presupone la duración de la percepción, o sea, que la percepción de cualquier configuración temporal tiene, ella misma, su configuración temporal" (Husserl, 1959, pág. 69). 
Los objetos viven en el presente. El futuro, por lo menos el más inmediato, se concreta en el ahora. Todo objeto es elaborado para el después, pues todo ser humano vive en el futuro: "Vivimos en el presente, en el punto actual, pero no existe primariamente para nosotros, sino que desde él, como desde un suelo, vivimos así el inmediato futuro" (Ortega \& Gasset, 2006, p. 229). Los objetos se elaboran para transitar del presente de su origen al futuro de la existencia como utilidad. Por ejemplo, las herramientas se producen para actuar en el tiempo, no solo para el ya de su existencia, su utilidad o función solo es posible en tanto duren en el tiempo. Nadie adquiere un producto que en el momento de su adquisición inmediatamente desaparezca. $\mathrm{Ni}$ siquiera los artículos desechables, una muestra actual de la corta vida de los objetos, ya que están hechos para cumplir una función en un después próximo. Pero no solo los objetos útiles, en el sentido clásico de suplir una necesidad física, también aquellos estéticos, por ejemplo una escultura, un cuadro, una composición musical, se comen literalmente el tiempo futuro:

entre más dure una obra en el tiempo, más valor estético gana para el mundo de la vida. De la duración de los objetos se desprenden conceptos como calidad, inversión y confianza, entre otros. De alguna manera los productos del diseño habitan en el futuro próximo, se prolongan en el tiempo en correlación con la duración de la percepción.

Ahora bien, si seguimos el pensamiento de Ortega y Gasset (2006), también debemos pensar en lo indescifrable del futuro, pues si bien es cierto que todo instante tiene en su haber su espera, su efectividad o materialidad, la existencia en el tiempo no está totalmente asegurada y esto produce incertidumbre:

\section{El dramatismo es la tensión sobre normal de nuestra alma, producida por also que se nos anuncia para el futuro, al que en cada instante nos aproximamos más. De suerte que la curiosidad, el temor o el apetito suscitado por ese algo futuro se multiplica, por sí mismo, acumulándose sobre cada nuevo instante (p. 113).}

Si aplicamos esto a la constitución de los objetos del futuro, entonces tenemos que pensar en la incertidumbre generada por los objetos en su duración y en su aparición en el tiempo no sido. Entre ellos y la existencia humana co-existe una complicidad pero también un abismo. Son compañeros inevitables en el presente vivido del sujeto, es casi imposible negarnos a co-habitar con ellos, están en todas partes, determinan nuestra existencia, se vuelven prótesis corporales y mentales en el hacer, el tener y el ser mismo de la humanidad. Pero a la vez son nuestros extraños, son lo otro, cerrados al mundo, sin horizontes intencionales. Su rostro nos dice que no comparten nuestra existencia, en su estructura finita se niegan a transitar al futuro y les obligamos a seguir persistiendo, los queremos en su presencia y nos negamos a su 
ausencia, ya sea por necesidad o por sentimientos, es decir, debido a su utilidad o su belleza.

De acuerdo con lo anterior, la temporalidad es esencial en la constitución de los objetos temporales. La experiencia de los objetos del pasado fusionada con la expectativa de su duración en el futuro, genera su identidad para la percepción humana. La identidad es precisamente su permanencia en el tiempo. Aunque sufran variaciones en cada época, siempre existe algo igual que evita la pérdida de esa identidad. Así, los primeros prototipos de los carros de Ford de 1903 son muy distintos a los modelos actuales, pero seguimos identificando un carro como tal. Así sean muchos los cambios, la identidad no se pierde en tanto dura en el tiempo, siempre existe algo esencial gracias a lo cual lo que es sigue siendo. En este sentido transita la pregunta de Husserl (1959):
¿Qué es en este caso el objeto idéntico? Es la serie de protoimpresiones y constantes modificaciones, una serie de semejanzas que produce configuraciones coincidentes de series de igualdad o distinción, pero siempre dentro de un marco de igualdad general: esta serie da una originaria conciencia de unidad (p. 164-165).

La constitución de los objetos se refleja en su identidad y esta es posible gracias a la esencia, a lo incambiable en ellos, a su morfología, es decir, el eidos. Lo que permanece es la idea de los objetos. Por ello, su constitución misma se genera en la conciencia y precisamente esta les da identidad cuando se nos aparecen en el mundo de la percepción. La conciencia es el escenario donde actúan y no pierden su ser. La duración de los objetos temporales evoca su unidad en el tiempo: "La identidad de los objetos temporales es, por consiguiente, un producto unitario constitutivo de ciertas posibles coincidencias identificadoras de rememoraciones" (Husserl, 1959, p. 164). La idea del objeto se refleja en su materialidad y esta indica el sentido que solo se constituye en la conciencia de la existencia de lo no sido, de lo que de alguna manera va a llegar a ser. Por tal razón, en la introducción a la Fenomenología del tiempo inmanente (Husserl, 1959) Ivvone Picard afirma:

\section{El futuro es un a priori, una dimensión originaria sin la que no existe la conciencia. La temporalidad se temporaliza a partir de él (y es por otra parte la única razón para que el movimiento del tiempo que pasa sea orientado, tenga una dirección, un sentido)" (p. 21).}

Los objetos se constituyen para el futuro, es más, se hacen verdaderos en esa dimensión que legitima el pasado. Sobre este particular, Picard declara: "El futuro es aquello por lo cual el pasado se torna verdadero" (Husserl, 1959, p. 24). En este sentido, los objetos como ideas pueden ser eternos, pero como materia, finitos, pues el tiempo los consume y desintegra. La muerte de la forma material se anuncia en su misma constitución. En el mundo físico, todos indican su propia existencia en 
el futuro, su propia muerte. Pero su esencia, su forma, su idea persiste en el tiempo, de modo que su identidad no puede estar en la materia sino en la idea respecto a ellos. Los objetos temporales empiezan a morir desde el momento en que se revelan al mundo por medio de su uso. Nacen condenados a su propia extinción, al olvido causante del fondo más profundo de los recuerdos. A medida que el tiempo fluye, más se acorta su futuro y se amplifica su pasado, hasta que en un momento determinado sencillamente se desvanecen de la existencia humana. ¿Cuántos objetos han desaparecido de la existencia? La respuesta a esta pregunta es imposible, pues si alguien la responde le da vida a los muertos del pasado al recordarlos y dejarín de estar en el olvido absoluto. Todo lo que en su momento fue nuevo, novedoso, en otro tiempo será viejo y todo cuanto ahora es viejo, en algún momento fue nuevo. He aquí el encanto del fluir temporal incrustado en los objetos del diseño.

\section{ENTRE LOS FANTASMAS DE LOS OBJETOS Y LOS OBJETOS FANTASMALES}

\section{Hablar de fantasmas es hablar de la presencia de la ausencia ${ }^{5}$. Esto} consiste en ser testigos de la existencia de los objetos, así su presencia no tenga realidad física sino fantaseada, una realidad en el pensamiento, el escenario original de los fantasmas. Los objetos materiales tienen formas de existir aun a pesar de su extinción en el mundo visible. La invisibilidad es la manera como sus fantasmas se expresan a la percepción. Extienden su plenitud material al mundo de la

${ }^{5}$ Esta reflexión la tomamos de JeanFrançois Lyotard (1996) al referirse al concepto de deseo. sensación y cuando se tornan ausentes, sus fantasmas se presentan al pensamiento, surgen a la reflexión. Estas maneras de aparecer y desaparecer la plantea Husserl (1959) con las siguientes palabras: "De modo que distinguimos apariciones perceptivas y apariciones fantaseadas; las últimas contienen un material aprehensivo, los 'fantasmas' (modificaciones actualizadoras de sensación); las primeras, sensaciones" (p. 155).

Para la sensación, el mundo se oculta y "desoculta" constantemente. Vivimos en un eterno fluir de intermitencias: en un momento aparecen unos objetos y en otro momento, otros aparecen y desaparecen. A diferencia de Dios, no podemos ver todo con simultaneidad. Cada mirada particulariza el mundo y lo reduce a 
manifestaciones fraccionadas. El

papel de los diseñadores consiste en "desocultar" nuevos objetos al mundo del uso y de la estética. Es restituir el mundo, su ojo lo amplifica a partir de lo invisible a lo visible, como lo expone Merlau-Ponty (1964): "El ojo es eso que se ha conmovido por cierto impacto del mundo y lo restituye a lo visible por los trazos de la mano" (p. 21). Quizá solo dejamos de estar con objetos, por lo menos conscientemente, en estado onírico. Dormir es una forma de ausentarse del mundo en general. Mientras para quien está despierto los objetos son intermitentes, para quien duerme son invisibles a su sensación, pues solo existen los habitantes de sus sueños y estos no tienen realidad física, sino fantasmal. El mundo se vuelve invisible cuando el sujeto cierra sus ojos como un par de "guillotinas" y los objetos desaparecen hasta que se hacen visibles al abrir los ojos y entrar al mundo de los fantasmas.
Los fantasmas de los objetos no son una ilusión, son parte del fundamento de la materialización de los mismos. El tiempo convierte a los objetos en fantasmas que divagan por el devenir del mundo físico. Es propio, entonces, intentar develar las maneras como estas invisibilidades se revelan al mundo de la sensación y al del diseño. El ser humano vive rodeado de objetos materiales que no aparecen solos, pues cada uno reúne múltiples posibilidades de ideas. En ellos habitan fantasmas y estos invocan la ausencia de lo que podría ser ese objeto pero no lo es: "lo imaginario es incoherente o improbable porque es imaginario y no es imaginario porque es incoherente, la menor partícula de lo percibido la incorpora inmediatamente a lo 'percibido', el fantasma más verosímil resbala por la superficie del mundo" (MeraluPonty, 1970, pág. 61). Los fantasmas residen en los objetos: cuando observamos un mosaico de fotografías y en él falta una de ellas, aparece el espacio "vacío". La pregunta que surge o por lo menos llama la atención, es la presencia de esa ausencia. Allí está el fantasma y reconocemos lo faltante cuando la totalidad evoca la carencia. Ese fantasma se expresa a la percepción como necesario en su ausencia.

Esta es una manera fácil, pero no la única, de identificar los fantasmas en los objetos. Cuando los diseñadores hacen una interpretación crítica de un producto, llámese silla, plato, edificación o cualquier otro que invoca significados intencionales, encontramos expresiones como: İcarece de un terminado más limpio!, iLe faltan determinantes gravitacionales que le den estabilidad! iEl color no es adecuado! iNo tiene estructura formal estética! Si analizamos estas apreciaciones -incluso cualquiera que se hace sobre un producto de diseño- siempre vamos a encontrar fantasmas que habitan en la subjetividad del crítico para expresarlas. Por ejemplo, la carencia del terminado limpio evoca en la mente la terminación limpia, su fantasma brota del pensamiento y se compara con el objeto en cuestión para hacer la afirmación. Lo mismo pasa con la estabilidad, el color, la forma estética. Cada uno de estos elementos existen pre-asignados en la formación de los expertos, viven latentes en sus mentes con el fin de volverse recursos críticos frente al mundo. De esta manera, en los objetos habitan fantasmas que esperan ser visibilizados en los diseños. En todo lo 
otros. De ese ideal, de ese prototipo que hala desde el futuro se infiere la inspiración seneradora de la creatividad, impulsadora de los genios del diseño. Algunos se aproximan más, otros, por el contario, se pierden en las apariencias y lo finito. Los objetos perfectos están perdidos en el tiempo y la intuición del diseñador los busca desesperadamente. "Estamos siempre en una retrospectiva del objeto perdido, incluso en el nivel del sentido, del lenguaje" (Baudrillard \& Nouvel, 2002, p. 27).

representando o configurado siempre existe lo representable, como fantasma. Así lo defiende Husserl (1959) al pensar en las representaciones: "En efecto, toda conciencia, toda 'sensación' en sentido más amplio, es algo 'perceptible' y 'representable'. Siempre tenemos de nuevo una conciencia que posee su posible contraparte en un fantasma" (p. 157).

La presencia fantasmal de las carencias en los objetos nos permite inferir que la perfección en ellos es una idea que nunca se perpetra con las manos ni brota de la subjetividad al mundo de la percepción. El objeto perfecto es una idea fantasmagórica que ronda permanentemente en los buenos diseñadores. Tal objeto adámico preexiste como fantasma en el mundo del diseño y en cada producto se develan rasgos, en unos más que en
La ausencia parcial, en lugar de aquella total, es otra manera como se evidencian los fantasmas en los objetos. En la actualidad se afirma que estos tienden a desaparecer, en tanto el principio básico parece ser conservar la función y perder tamaño. Al menos, así lo reconocen Baudrillard y Nouvel (2002, p. 52):

\section{Todo lo real está listo para desaparecer, no pide otra cosa y en el dominio de la arquitectura, y más que en la arquitectura, en el dominio del diseño en general, estamos en esta estética del 'sacrificio'. Diría que el sacrificio de lo visual, no sé hasta dónde, pasa por la miniaturización, por un dominio cada vez mayor de la materia y la propia materia se reduce cada vez más a su más simple expresión.}

Los elementos que desaparecen, que se sacrifican de los objetos, siguen existiendo en ellos como fantasmas vivos, en el pensamiento colectivo de los usuarios. El estéreo (equipos de audio), otrora de un metro de alto por cuarenta centímetros de ancho, se mantiene vivo, primero en los transistores o radios, luego en los Apple y en las memorias USB. En estas miniaturizaciones existen los elementos que se han sacrificado, en tanto la función, el sonido, permanece viva. Esto pasa en arquitectura, cuando comparamos la casa familiar antigua con los apartamentos actuales, donde se siguen conservando los fantasmas de la niñez de la casa tradicional. La moda es intentar desaparecer los objetos, es decir, quedarnos solo con sus fantasmas.

El diseño tiende a desaparecer los objetos. Vivimos un tiempo en que el mundo se reduce, no en importancia ni calidad, sino en materia. Vivimos una época de desmaterialización de los objetos, sacrificamos lo perceptible de ellos con el fin de generar 
significados de simplicidad,

lo cual evoca menos trabajo. Para

tal fin, inventamos múltiples formas

de desaparecerlos. "La estética de la desaparición habría que entenderla bajo el término... es verdad que hay mil formas de desaparecer" (Baudrillard \& Nouvel, 2002, p. 49). Podemos esconder los objetos, reducir sus características materiales, pulverizarlos o simplemente invisibilizarlos a la percepción. Lo que queda de la reducción, de la pulverización, los rastros o huellas de la invisibilización, no son más que los sedimentos fantasmales hechos presentes como materia ausente. Si la desmaterialización implica la destrucción, vivimos una época en que el deshacer predomina sobre el hacer. Esta aniquilación parece ser el imperativo presente del diseño, algo que en últimas suple las perversiones más íntimas del diseñador. Así es formulado por Baudrillard y Nouvel al referirse al arte y la arquitectura: "en el arte y en la arquitectura hay una búsqueda de límites y un placer de la destrucción" (p. 40).

La búsqueda de la desmaterialización o la miniaturización parece conducir a la invisibilidad del diseño en el mundo físico. Esta es la tercera postura frente a la seneración de objetos fantasmales y aquí tenemos que salirnos de la singularidad de los objetos y concentrarnos en los contextos donde ellos son unidades de sensación. En esos ambientes, o sistemas, para el caso de Baudrillard, la colectividad crea significados de los objetos, ya sea en la casa, la vecindad, la ciudad, el país y aun en el mundo visto en su totalidad. Este tema exige un amplio desarrollo, por el momento, cabe referenciarlo como otra manera de desparecer los objetos. Al respecto, convocamos a Barthes (1993): "Significar quiere decir que los objetos no transmiten solamente información, sino también sistemas estructurados de signos, es decir, esencialmente sistemas de diferencias, oposiciones y contrastes". (p. 246).

En los ambientes, entonces, hay objetos invisibilizados, ya sea por su sencillez o por su existencia innecesaria en el contexto donde se determinan. En la relación de las partes con el todo, hay cosas que pierden su condición de ser percibidas, precisamente por su singularidad dentro del sistema. En la vida cotidiana se escucha con frecuencia la expresión lyo nunca había visto eso! Pero eso siempre ha estado allí. La singularidad desaparece ante la percepción de la totalidad. Este es un acto de magia que se activa y desactiva en la conciencia humana. Sobre el particular es pertinente volver a Baudrillard y Nouvel (2002):

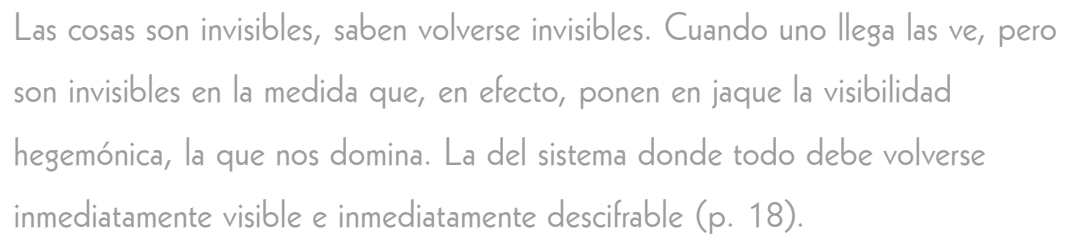


Un cuarto factor que podemos enunciar en la generación de objetos fantasmas son los productos de las nuevas tecnologías, sobre todo en las telecomunicaciones. Cuando nos enfrentamos a un celular, a un computador e incluso al televisor, nos encontramos con dos realidades, una virtual y otra material, que se dan de forma alternada a la conciencia humana. Lo presentado virtualmente anula lo material, pues cuando vemos lo que nos ofrece el televisor, esto se sobrepone al interés por el aparato en sí. Cada elemento hace invisible al otro a la percepción humana, lo convierte en un fantasma. No tiene sentido sentarse a ver el televisor (por lo menos no para los usuarios, sí para los diseñadores), la estructura física desaparece con las imágenes proyectadas, estas nos atrapan y la conciencia es mera conciencia de lo virtual. Esto mismo pasa con los celulares y los computadores, aunque en grados diferentes. En general, los objetos conservan esta dinámica: una cosa es lo que nos muestran fantasmalmente y otra lo que son en sentido físico. A esta reflexión cabe abonar la existencia misma de la virtualidad, la cual solo existe a la conciencia cuando aparece a la percepción. Una vez desaparece, al apagar el equipo, se invisibiliza y se pierde no solo a los ojos sino al mundo de la materia. Sin embargo, una vez encendemos el computador esperamos que cuanto dejamos allí todavía aparezca a nuestra vista. Su presencia no es del tiempo, ni pasado ni futuro, sino de las vivencias humanas. El resto son fantasmas.

Un ejemplo no igual aunque similar son los espejos. Estos duplican la existencia del mundo para la percepción. El mundo se hace más amplio cuando estamos frente a ellos, pero curiosamente, los objetos les son invisibles, les son fantasmas. Los objetos no se pueden mirar, son ciegos para sí mismos, son cerrados a la realidad, pero el espejo redobla su existencia, los presenta a la percepción no desde el plano físico sino desde su reflejo, para convertir así la realidad objetual en fantasmal. En este sentido, el orden de los espejos como objeto del diseño representa un símbolo de poder que genera la sensación de habitar varios mundos y contribuye así al desarrollo de la conciencia, según explica Baudrillard (1981): "Digamos, en términos más generales, que el espejo, objeto de orden simbólico, no solo refleja los rassos del individuo, sino que acompaña en su desarrollo el desarrollo histórico de la conciencia individual" (p. 21). El espejo surgió como necesidad, producto de la egolatría esto es una hipótesis - hasta convertirse en un acompañante fiel de la humanidad y su sistema de objetos. Nació como necesidad del cuerpo humano de verse a sí mismo, o por lo menos de reconocerse en el mundo visual, de acuerdo con la afirmación de Merleau-Ponty (1964): "Como todos los demás objetos técnicos, como las herramientas, como los signos, el espejo ha surgido en el circuito abierto del cuerpo vidente al cuerpo visible" (p. 26). Los espejos recrean los fantasmas, los hacen visibles al mundo de la percepción, pero intocables al mundo físico. También expresan dos realidades alternas: lo que se refleja en ellos y la materialidad que los acompaña. 
Otro fenómeno de ocultación se da en las vitrinas. Estas permiten ver sin tocar, su esencia está precisamente en desparecer a la sensación para que la mirada penetre su ser y deje ver su interior. Se hacen fantasmas frente a la conciencia humana pues el vidrio tiene esta propiedad, la de aparecer como fantasma al mundo de la vida, permite traspasar la materia con la mirada, priva al ser humano de su intimidad, lo hace público, publicita los productos. Las vitrinas invisibilizan el mundo. Bajo esta dinámica y pensando en términos futuristas, la nada es el escenario hacia donde tiende el producto del diseño, es el lugar de lo ininteligible, el lugar sin lugar, la muerte absoluta de los objetos, el sinsentido de la existencia: "La nada es el lugar del secreto, el lugar donde las cosas pierden su sentido, donde se desidentifican, no solo donde adquirirían todos los sentidos posibles, sino donde permanecen verdaderamente, en cualquier parte, ininteligibles". (Baudrillard \& Nouvel, 2002, pág. 30).
La tendencia de los objetos del diseño es hacerse ellos mismos fantasmas, ya sea en su desmaterialización, en su miniaturización, en la evocación de las ausencias, en la pérdida de identidad en la totalidad o en la virtualidad. La nada llama en lo más íntimo de los usuarios a la minimización del mundo de los útiles y esto, desde la reflexión, produce sentimientos encontrados, tales como vértigo, nostalgia y hasta delirio. El vértigo de saberse desubicado, de no conocer con precisión el camino del diseño, la ruta que muestre la resurrección de los objetos, el producido por la conciencia de la nada a la cual nos conducen los fantasmas de estos. Los objetos generan nostalgias, ya sea por la función o por la composición estética. Damos vida en el presente a aquellos del pasado, con el fin de revivirlos en el futuro. Esto, en últimas, causa delirio, dada la incertidumbre que inquieta a los diseñadores y los impulsa a buscar el objeto perfecto e ideal. Pero el genio del mal, al parecer, siempre aparece en los procesos que llaman desde lo profundo de los objetos a no dejarse perpetrar por las ideas: "El genio del mal está detrás de todo objeto" (Baudrillard \& Nouvel, 2002, pág. 25). El papel del diseño es seguir desentrañando la encrucijada del futuro para situar el presente de los objetos, como afirma Merleau-Ponty (1964):

\section{Es necesario que el pensamiento de ciencia - pensamiento de sobre vuelo, pen- samiento del objeto en general- se vuelva a situar en un 'hay' previo, en el sitio, en el suelo del mundo sensible y del mundo trabajado tal como está en nuestra vida, para nuestro cuerpo" (p. 11)}

\section{CONCLUSIONES}

Al inicio de este trabajo proponíamos la siguiente hipótesis: si en los productos del diseño se encuentran encapsulados el tiempo sido y el no sido, entonces, en la interpretación de los objetos, está la sedimentación del futuro. Ahora podemos afirmar tal enunciado en coherencia con estas tesis:

Primera. La única existencia temporal real es el instante vivido. En este seda el ahora retencivo y el ahora propencivo, es decir, en el instante se funden el pasado en forma de recuerdo y el futuro en forma de espera. 
Segunda. La experiencia de los objetos solo es posible en el presente.

De tal forma, ellos coexisten con las vivencias humanas. Por tal razón podemos hablar de objetos vivenciales, los cuales poseen implicaciones de los objetos pasados y de aquellos del futuro. En el objeto se encuentra el tiempo vivido y el no vivido.

Tercera. El ser humano puede capturar los objetos, y en general el mundo, solo por esbozos. Así, para identificar los objetos e intervenir en ellos necesita los sentidos -que son sus límites- y la razón. Los objetos nacen incipientes y la humanidad, evolutiva e histórica, da forma a los productos del diseño.

Cuarta. Los objetos no son más que la individualización del mundo hecha por los seres humanos a partir de la singularización de sus sentidos. Esta particularización se fundamenta en la función, la cual surge de las necesidades básicas o de la estética, de la búsqueda del disfrute o de la lúdica. Entonces, tenemos dos formas de experiencia, una útil y otra frente a lo bello.

Quinta. Los objetos para el diseño son fragmentos del mundo en que el ser humano ha puesto intencionalidad, por medio de significados. Por ello, hay objetos simples y compuestos. El papel del diseño es remover los elementos móviles de estos últimos.

Sexta. Los objetos no son solo existencias materiales. En cada uno habitan ideas creadas por la historia y estas existen empaquetadas en teorías científicas o precientíficas que se revelan en ellos. De ahí el papel de la pedagogía del diseño.

Séptima. Los objetos tienen la intencionalidad del después, pues no se diseña para el ya sino para un futuro próximo o lejano. De su tiempo de vida depende el concepto de identidad, el cual es más fuerte en tanto coexiste en el mundo de la percepción y en el de los recuerdos.

Octava. El ser humano encuentra una encrucijada cuando se enfrenta a los objetos. Son sus acompañantes fieles, pero también son extraños creados en el tiempo que evocan un mundo ajeno a la manera como el sujeto vive y los vive.

Novena. En la actualidad, los objetos tienden a la minimización de su extensión material y han empezado a desaparecer, lo cual se manifiesta de diferentes formas: desmaterialización, productos tecnológicos, espejos y vitrinas. Estos son ejemplos de esa ausencia de la presencia, es decir, de los fantasmas de los objetos que existen o de los que no existen como realidad física, sino como idealidad en el futuro. 
Bachelard, G. (1999). La intuición del instante. México, México: Fondo de -cultura Económica.

Barthes, R. (1993). La aventura semiológica. Barcelona, España: Paidós.

Baudrillard, J. (1981). El sistema de los objetos. Madrid, España: Siglo Veintiuno.

Baudrillard, J., \& Nouvel, J. (2002). Los objetos singulares. Arquitectura y filosofía.

Buenos Aires, Argentina: Fondo de Cltura Económica.

Bollnow, O. (1969). Hombre y espacio. Barcelona, España: Labor.

Husserl, E. (1959). Fenomenología de la conciencia del tiempo inmanente. Buenos Aires, España: Nova.

Husserl, E. (1984). Ideas Relaltivas a una fenomenología pura y una filosofía fenomenológica. Libro II: Investigaciones fenomenológicas sobre la constitución.

México, México: Universidad Autónoma de México.

Husserl, E. (1995). Investigaciones lógicas II. Barcelona, España: Altaya. 
Levinas, E. (2004). La teoría fenommenológica de la intuición. Salamanca, España: Sígueme.

Lyotard, J.-F. (s.f.). ¿Por qué filosofar? Barcelona, España: Altaya.

Meralu-Ponty, M. (1970). Lo invisible y lo visible. Barcelona, España: Seix Barral.

Merlau-Ponty, M. (1964). El ojo y el espíritu. Buenos Aires, Argentina: Paidós.

Ortega y Gaset. (2006). ¿Qué es la filosofía? Madrid, España: Espasa Calpe.

Picard, I. (1959). el tiempo en Husserl y Heidegger. Introducción a la fenomenología del tiempo inmanente. En Husserl Edmund, Fenomenología del tiempo inmane te. Buenos Aires, España: Nova.

Vanegas García, J. H. (Abril de 2008). Del lenguaje objetual al diseño industrial. Disertar, 1(1), 49-82.

Zubiri, X. (1944). Naturaleza, historia, Dios. Buenos Aires, Argentina: Poblet. 